\title{
BMC International Health and Human Rights Reviewer Acknowledgement 2014
}

Giulia Mangiameli

\section{Contributing reviewers}

The editors of BMC International health and human rights would like to thank all our reviewers who have contributed to the journal in Volume 14 (2014).

Seye Abimbola

Nigeria

Elizabeth Abu-Haydar

USA

Philip Adongo

Ghana

Vishal Aggarwal

UK

Kingsley Agho

Australia

Mohamed Azmi Ahmad Hassali

Malaysia

Ameena Ahmed

USA

Moses Aikins

Ghana

George Annas

USA

Jennifer Anyanti

Nigeria

Odwa Atari

Canada

Chamila Thushari Attanapola

Norway

Edwinah Atusingwize

Uganda
Jenita Baruah

India

Gajananda Bhandari

Nepal

Ilse Blignault

Australia

Marleen Bosmans

Belgium

Stine Hellum Braathen

Norway

Robert Brooks

Australia

Garrett Brown

UK

Justine Bukenya

Uganda

Alasdair Cochrane

UK

Claudia Cappa

USA

Arachu Castro

USA

Rose Chapman

Australia

Jeremiah Chikovore

South Africa
Cari Clark

United States Minor Outlying Islands

Jocalyn Clark

Bangladesh

Todd Coleman

Canada

Anne Lia Cremers

Netherlands

Joanne Csete

USA

Lucia D'Ambruoso

UK

Eugene Kofuor Maafo Darteh

Ghana

Bregje De Kok

UK

Richard Elliott

Canada

Charli Eriksson

Sweden

Kathryn Falb

USA

Allison Friedman

USA

Eric Friedman

USA

Correspondence: giulia.mangiameli@biomedcentral.com

BioMed Central, Floor 6, 236 Gray's Inn Road, London WC1X 8HB, UK 
Ines Fronteira

Portugal

Ana Gama

Portugal

Abebaw Gebeyehu

Ethiopia

Tesfay Gebregzabher Gebrehiwot

Ethiopia

Nikoletta Giatras

UK

Roger Gibson

Jamaica

Amanda Glassman

USA

Oliver Gruebner

USA

José Hagan

USA

Rachel Hammonds

Belgium

Sandya Hewamanne

USA

Shashivadan Hirani

UK

Terri Hyde

USA

Yulia Izati

Indonesia

Zarina Nahar Kabir

Sweden

Soumik Kalita

India

Mariano Kanamori Nishimura

USA

Godfrey Kangaude

Malawi

Kristi Kenyon

Canada

Kaveh Khoshnood

USA

Vincent Z. Kuuire

Canada

Yiannis Kyratsis

UK
Greg Layther

UK

Kenneth L Leonard

USA

Leslie London

South Africa

Svjetlana Lozo

USA

David Lubogo

Uganda

Roger Magnusson

Australia

Mats Malqvist

Sweden

Lochner Marais

South Africa

Maria Rosario Martins

Portugal

Peter Ebongue Mbondji

Cameroon

Gitau Mburu

UK

Margaret Mcconnell

USA

Anita Mcgahan

Canada

Matthew Mckenna

USA

Benjamin Meier

USA

Mohamed Izham Mohamed Ibrahim

Malaysia

Jose Moraes

Brazil

Alejandro Moreno

USA

Alexandra Muller

South Africa

Haruka Nakada

Japan

Brett Nelson

USA

Elizabeth Newnham

Australia
Benjamin Nganda

Kenya

Justice Nonvignon

Ghana

Noah Novogrodsky

USA

Jerry Okal

Kenya

Gorik Ooms

Belgium

Kwaku Oppong Asante

Ghana

Maria José Osis

Brazil

Rene Osorio

USA

Yin Paradies

Australia

Lauren Paremoer

South Africa

Parveen Parmar

USA

Robert Peck

Tanzania

Nasheeta Peer

South Africa

Emma Plugge

UK

Miriam Potocky

USA

Lisa Reynolds

UK

Adam Richards

USA

Esther Richards

UK

Richard Rinehart

USA

Giuliano Russo

Portugal

Elizeus Rutebemberwa

Uganda

Ricardo Rüttimann

Argentina 
Shepherd Shamu

Zimbabwe

Geordan Shannon

UK

Mukta Sharma

Thailand

Kenneth Sherr

USA

Sara Shuman

USA

Teresa Spadea

Italy

Annie Sparrow

USA

Allison Squires

USA

Sarah Jane Steele

Canada

Alistair Stewart

New Zealand

Miriam Taegtmeyer

UK
Augustinus Ten Asbroek

UK

Mikiko Terashima

Canada

Lil Tonmyr

Canada

Lyndal Trevena

Australia

Alexander Tsai

USA

Remco Van De Pas

Belgium

Johannes Van Rooyen

South Africa

Murali Dhar Vemuir

India

Sridhar Venkatapuram

UK

Gwenaelle Vidal-Trecan

France

Kerri Viney

Australia
Abdul Wajid

USA

Joyce Wamoyi

Tanzania

Daniel Edward Wight

UK

Erin Wilson

USA

William Wolfe

USA

Junqing Wu

China

Qingwen Xu

USA

Katherine Yun

USA

Cathy Zimmerman

UK 